\section{FRI0128 A NOVEL FORMULATION OF CT-P13 (INFLIXIMAB BIOSIMILAR) FOR SUBCUTANEOUS ADMINISTRATION: 1-YEAR RESULTS FROM A PART 1 OF PHASE I/III RANDOMIZED CONTROLLED TRIAL IN PATIENTS WITH ACTIVE RHEUMATOID ARTHRITIS}

DaeHyun Yoo ${ }^{1}$, Janusz Jaworski ${ }^{2}$, Ewa Matyska-Piekarska ${ }^{2}$, Svitlana Smiyan $^{3}$, Delina Ivanova ${ }^{4}$, Agnieszka Zielinska $^{5}$, Eve-Kai Raussi ${ }^{6}$, Anastas Batalov ${ }^{7}$, Sangjoon Lee ${ }^{8}$, Jeehye Suh ${ }^{8}$, Noori Han ${ }^{8}$, Rene Westhovens ${ }^{9} .{ }^{1}$ Hanyang University for Rheumatic Diseases, Seoul, Korea, Rep. of (South Korea); ${ }^{2}$ REUMATIKA-Centrum Reumatologii NZOZ, Warszawa, Poland; ${ }^{3}$ State Higher Educational Institution "I.Ya. Horbachevskyi Ternopil State Medical University of $\mathrm{MOH}$ of Ukraine", Ternopil, Ukraine; ${ }^{4}$ Diagnostic-Consultative Center

Aleksandrovska EOOD, Sofia, Bulgaria; ${ }^{5}$ Medycyna Kliniczna, Warszawa, Poland ${ }^{6}$ North Estonia Medical Centre Foundation, Tallinn, Estonia; ${ }^{7}$ Medical University Plovdiv, University Hospital Kaspela, Clinic of Rheumatology, Plovdiv, Bulgaria; ${ }^{8}$ Celltrion, Inc., Incheon, Korea, Rep. of (South Korea); ${ }^{9}$ University Hospital KU Leuven, Leuven, Belgium

Background: Efficacy and safety of a new subcutaneous (SC) formulation (CT-P13 SC) up to Week 30 were comparable with intravenous (IV) formulation (CT-P13 IV) in both patients with rheumatoid arthritis (RA) [1] and Crohn's disease [2].

Objectives: This report is to further investigate pharmacokinetics, efficacy and overall safety of CT-P13 SC in patients with RA throughout the 1year treatment period.

Methods: Patients with active RA (presence of 6 or more swollen and tender joints [of 28 assessed], and serum C-reactive protein [CRP] concentration $>0.6 \mathrm{mg} / \mathrm{dL}$ ) were treated with CT-P13 IV at Weeks 0 and 2, and were randomized for continuation with CT-P13 IV or SC administration at Week 6. The IV cohort received CT-P13 IV $3 \mathrm{mg} / \mathrm{kg}$ every 8 weeks and the SC cohorts received CT-P13 SC $90 \mathrm{mg}, 120 \mathrm{mg}$ or 180 $\mathrm{mg}$, respectively, every 2 weeks up to Week 54. Pharmacokinetics blood samples were collected before study drug administration at each visit and drug levels were determined by electrochemiluminescent assay. Efficacy parameters including DAS28 and ACR criteria and overall safety were evaluated.
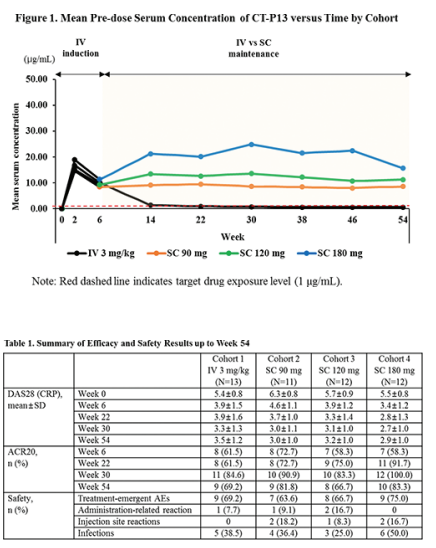

Results: A total of 50 patients were enrolled, of whom 48 patients were randomly assigned at Week 6 into 4 cohorts (1:1:1:1 ratio). The mean Ctrough (pre-dose serum concentration of CT-P13 before next dose injection) of SC cohorts throughout the study visits were higher than those of IV cohort after randomization at Week 6. Ctrough levels increased with SC dose and were sufficiently higher than the target therapeutic concentration $(1 \mu \mathrm{g} / \mathrm{mL})$ throughout the study period (Figure 1). Overall, the efficacy results of CT-P13 SC up to Week 54 were comparable to those of CT-P13 IV. Disease improvement by DAS28 (CRP) and ACR20 were comparable across all 4 cohorts, regardless of the route of administration or dosage of CT-P13 (Table 1). The safety profiles which occurred after study drug administration at Week 6 in SC cohorts were generally comparable to those of IV cohort and appeared similar to those previously reported for IV infliximab [3]. All injection site reactions were grade 1 or 2. No malignancy or death was reported (Table 1).

Conclusion: The results from 1-year treatment suggest similar efficacy and safety of CT-P13 SC to CT-P13 IV in RA. The mean serum concentration in all SC cohorts consistently exceeded the threshold of target therapeutic concentration. These results show that the novel SC formulation of CT-P13 may enhance treatment options for use of infliximab biosimilar by providing high consistency in drug exposure.

\section{REFERENCE:}

1 Westhovens et al., Annals of the Rheumatic Disease 2018:77:315

2 Schreiber et al., Gastroenterology 2018;154(6):S-1371.

3 Yoo et al., Arthritis Research 18:82.

Disclosure of Interests: DaeHyun Yoo Grant/research support from: Celltrion, Inc., Consultant for: Celltrion, Inc., Janusz Jaworski Grant/research support from: Celltrion, Inc., Ewa Matyska-Piekarska Grant/research support from: Celltrion, Inc., Svitlana Smiyan Grant/research support from: Celltrion, Inc., Delina Ivanova Grant/research support from: Celltrion, Inc, PPD, Quintiles, Egis Pharmaceuticals, and Pfizer., Agnieszka Zielinska Grant/research support from: Celltrion, Inc., Eve-Kai Raussi Grant/research support from: Celltrion, Inc. and Board of Estonian Society of Rheumatology, Anastas Batalov: None declared, SangJoon Lee Shareholder of: Celltrion, Inc., Employee of: Celltrion, Inc., JeeHye Suh Employee of: Celltrion, Inc., Noori Han Employee of: Celltrion, Inc., Rene Westhovens Grant/research support from: Bristol-Myers Squibb, Consultant for: Celltrion, Galapagos-Gilead

DOI: 10.1136/annrheumdis-2019-eular.1094

\section{Rheumatoid arthritis - non biologic treatment}

\section{FRI0129 THE BTK INHIBITOR, FENEBRUTINIB, EFFECTIVELY MODULATES B AND MYELOID CELL BIOLOGY IN RHEUMATOID ARTHRITIS PATIENTS}

Alyssa Morimoto $^{1,1}$, Julie Rae ${ }^{1}$, Leslie Chinn ${ }^{2}$, Nandhini Ramamoorthi ${ }^{3}$, Olivia Hwang ${ }^{1}$, Alexandra Ward ${ }^{4}$, D. James Haddon ${ }^{1}$, Caroline Looney ${ }^{1}$,

Rupal Desai ${ }^{5}$, Balazs Toth ${ }^{6}$, Katie Tuckwell ${ }^{7}$, Michael J. Townsend ${ }^{3} .{ }^{1}$ Genentech/ Roche, OMNI Biomarker Development, South San Francisco, United States of America; ${ }^{2}$ Genentech/Roche, Clinical Pharmacology, South San Francisco, United States of America; ${ }^{3}$ Genentech/Roche, Biomarker Discovery OMNI, South San Francisco, United States of America; ${ }^{4}$ Genentech/Roche, Biomarker Operations, South San Francisco, United States of America; ${ }^{5}$ Genentech/Roche, Companion Diagnostics, South San Francisco, United States of America; ${ }^{6}$ Genentech/Roche, Biometrics, South San Francisco, United States of America; ${ }^{7}$ Genentech/Roche, Early Clinical Development, South San Francisco, United States of America

Background: Bruton's tyrosine kinase (BTK) plays an essential role in $B$ cell development. BTK acts downstream of the B cell receptor in B cells, and $\mathrm{Fc}$ receptor signaling in myeloid cells, pathways thought to be involved in the pathogenesis of RA. Fenebrutinib (FEN) is a non-covalent small molecule inhibitor of BTK with greater than 100-fold selectivity relative to other kinases ${ }^{1}$.

Objectives: To characterize the mechanistic effects of FEN on B and myeloid cell biology and determine the potential of biomarkers to predict response to FEN treatment in patients with RA.

Methods: The ANDES study included RA patients (pts) on background methotrexate (MTX) with inadequate response to prior MTX $(n=480$, Cohort 1, MTX-IR) or anti-TNFs ( $\mathrm{n}=98$, Cohort 2, TNF-IR). Cohort 1 pts were randomized to receive PBO, adalimumab (ADA) $40 \mathrm{mg}$ Q2W, or FEN $50 \mathrm{mg} \mathrm{QD}, 150 \mathrm{mg}$ QD or $200 \mathrm{mg}$ BID. Cohort 2 pts were randomized to receive PBO or FEN $200 \mathrm{mg}$ BID. Clinical efficacy was assessed based on the proportion of pts achieving ACR50 at week 12 Pts for whom samples were available were assessed for levels of rheumatoid factor (RF), total IgM and IgG, CCL4, CXCL13, CRP and IL6. Results: Primary study results are reported separately. Overall, treatment with FEN caused significant reductions in RF (by week 12) and total IgM and IgG (at weeks 4-12) relative to PBO (Table 1). Early and sustained reductions of the B cell chemokine CXCL13 and the myeloid-enriched biomarker CCL4 were observed with FEN or ADA relative to PBO by week 1. CRP levels were significantly reduced with $200 \mathrm{mg} \mathrm{BID} \mathrm{FEN} \mathrm{by} \mathrm{week} 8$, and with ADA by week 2 relative to PBO. By week 12, there was a trend toward lower IL6 levels with FEN treatment relative to PBO, whereas ADA significantly reduced IL6 levels by week 1 relative to PBO in MTX-IR pts. In TNF-IR patients, IL6 was significantly reduced by FEN treatment by week 12 relative to PBO. PK-PD relationships were observed for multiple B and myeloid cell biomarkers. No single biomarker at baseline was associated with clinical response (at 12 weeks) in MTX-IR and TNF-IR pts. However, greater baseline RF titers were associated with increased FEN clinical response in TNF-IR pts. Conclusion: FEN treatment resulted in strong pharmacodynamic effects on multiple biomarkers of $B$ and myeloid cell biology in RA. This included reductions in total $\operatorname{lgM}$ and $\lg$ that were not observed with ADA. Conversely, ADA reduced IL6 and CRP faster than FEN, highlighting key mechanistic differences between FEN and ADA. Baseline RF titer was associated with FEN clinical response in the more refractory TNF-IR 